\title{
Improved Assembly Processes for the Quartz Digital Accelerometer Cantilever
}

\author{
Albert M. Romero \\ Sensor Components Division \\ Charles T. Gebert \\ Prototype Dèvelopment and Evaluation Division \\ Sandia National Laboratories \\ Albuquerque, NM 87185
}

\begin{abstract}
This report covers the developmert of improved assembly processes for the Quartz Digital Accelerometer cantilever. In this report we discuss improved single-assembly tooling, the development of tooling and processes for precision application of polyimide adhesive, the development of the wafer scale assembly procedure, and the application of eutectic bonding to cantilever assembly.
\end{abstract}




\section{Acknowledgments}

The authors acknowledge and thank the following persons for their contributions:

Bill Kass, Div. 2533, for his guidance on the requirements of the Quartz Digital Accelerometer (QDA) and for having samples of QDAs fabricated by each technique described in this report.

Joyce Torres of Ktech, for her conscientious assembly of cantilevers with several iterations of tooling, and for performing the slew tests on the assemblies.

Paul Vianco, Div. 2532, for his thorough studies and careful development of the eutectic bond process.

R. Rossmeisl of GEND, for his critique of the tooling and processes:

J. Staudte of XECO, for his consultation on various aspects of the tuning forks and wafers affecting tooling and bonding. 


\section{Contents}

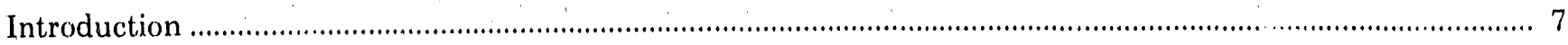

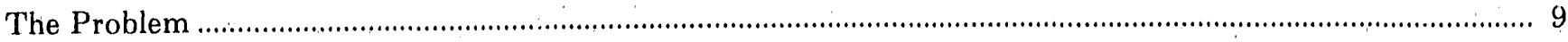

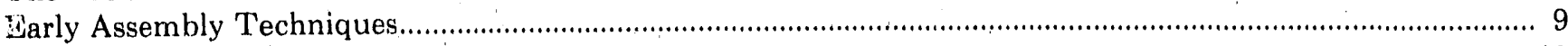

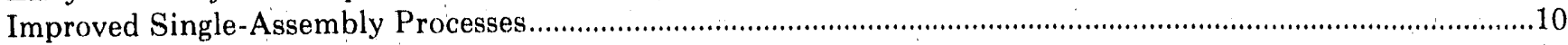

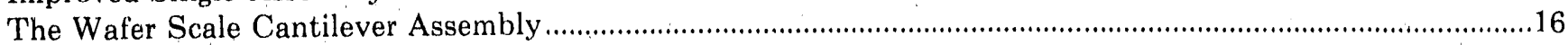



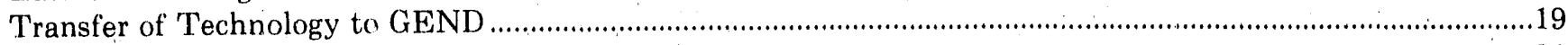

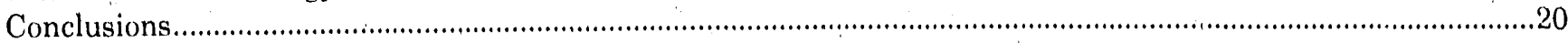

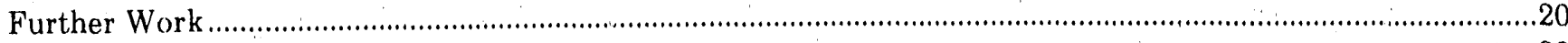

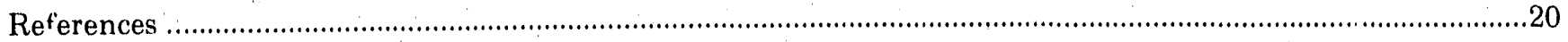

\section{Figures}

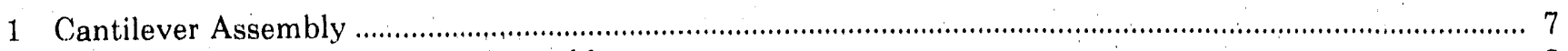

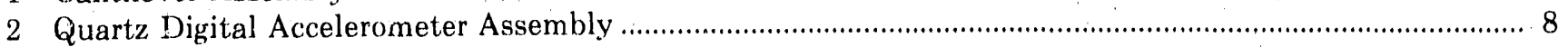

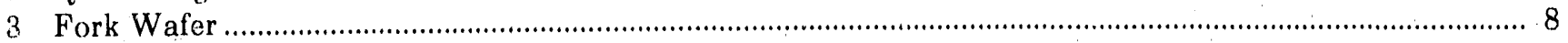

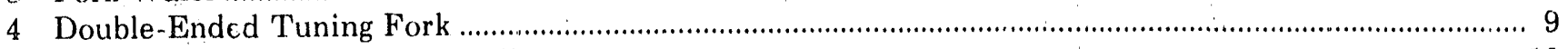

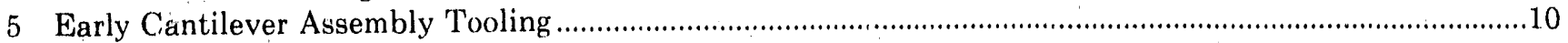

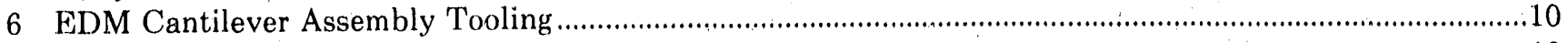

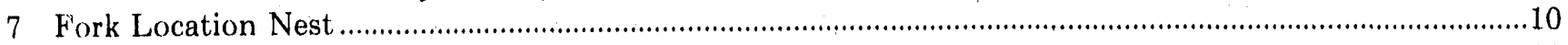

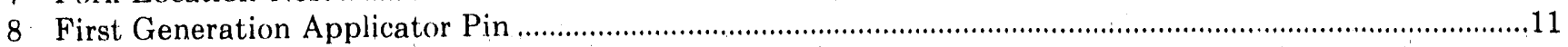

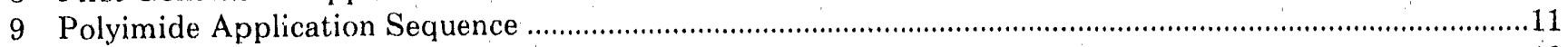

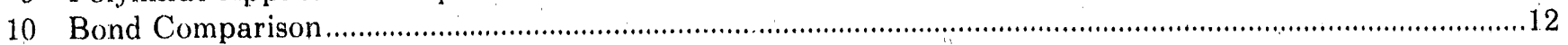

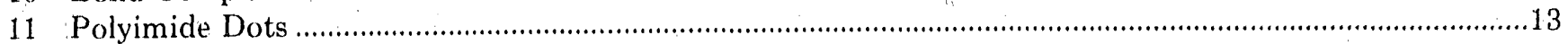

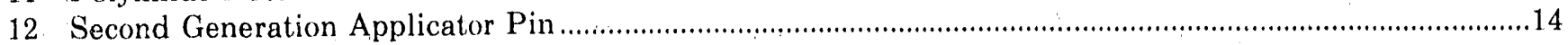

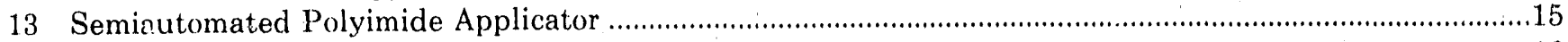

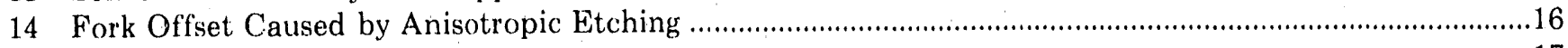



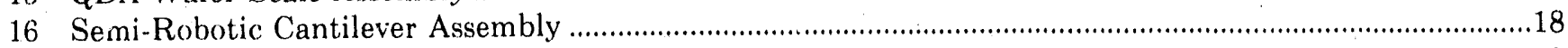

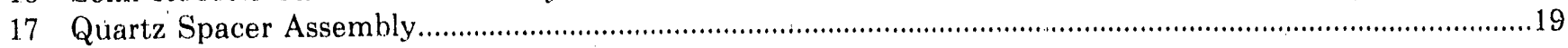




\section{Improved Assembly Processes for the Quartz Digital Accelerometer Cantilever}

\section{Introduction}

This report furnishes highlights of the cantilever assembly process development effort for the Quartz Digital Accelerometer (QDA). Our goal was to design the tooling and to define the processes required to produce a QDA cantilever assembly, reducing misalignments and stresses until the effect on cantilever performance became negligible. A further, more basic goal, was to develop precise, repeatable techniques that would apply in general to the assembly of miniature components.

\section{Description}

The QDA cantilever assembly consists of two miniature quartz, double-ended tuning forks separated by spacers at each end (Figure 1) and cantilevermounted in a ceramic frame. One spacer is used for mounting the assembly to the frame; the other serves as the proof mass that sensitizes the device to an acceleration field. After the QDA is assembled in the frame, electrical connections are made to the two forks, and the package is vacuum-sealed (Figure 2).

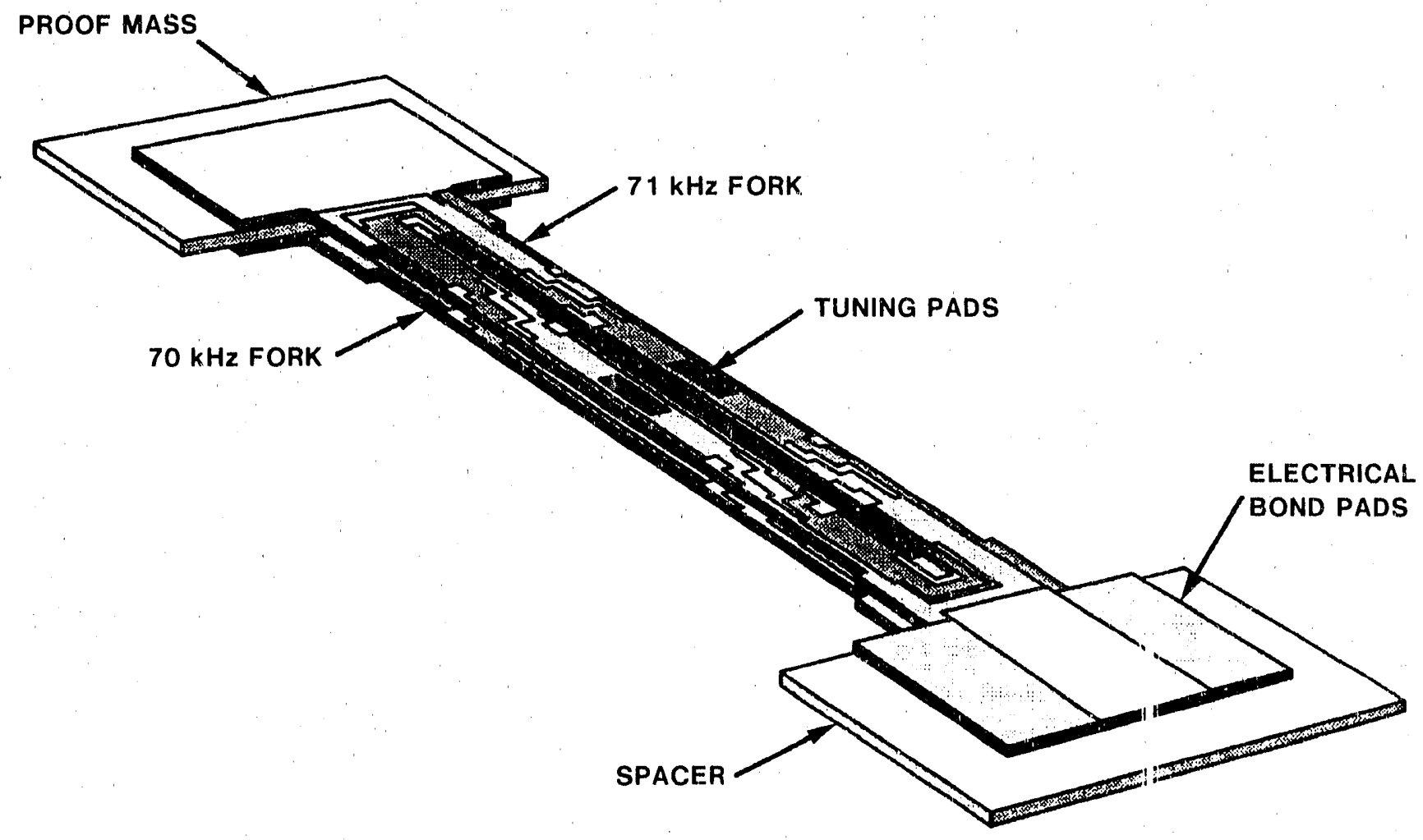

Figure 1. Cantilever Assembly 


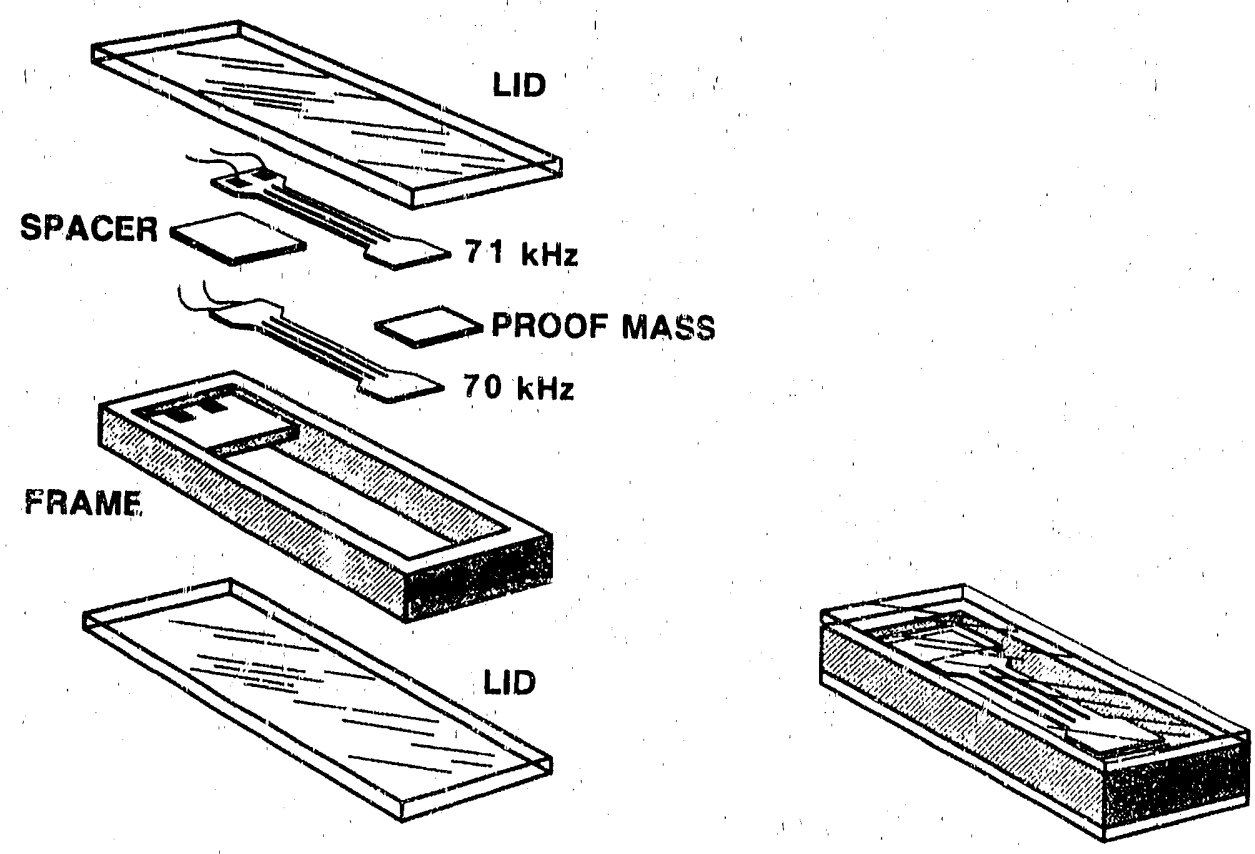

Figure 2. Quartz Digital Accelerometer Assembly

The two tuning forks are designed to resonate at frequencies $1 \mathrm{kHz}$ apart, one at $70 \mathrm{kHz}$ and the other at $71 \mathrm{kHz}$. In operation, an acceleration field causes the cantilever assembly to deflect. This alters the individual frequencies of the forks, increasing the one in tension and decreasing the one in compression. The resultant change in the difference of the frequencies is proportional to the acceleration field, thus permitting acceleration to be measured. The QDA inay have a sensitivity of $1 \mathrm{~Hz} / \mathrm{g}$ to $4 \mathrm{~Hz} / \mathrm{g}$, depending on the size of the proof mass.

The tuning forks are fabricated from 6-mil-thick wafers of quartz. Polished z-cut wafers are plated with chrome and gold and then chemically milled and patterned with electrodes using photolithographic techniques. Typical tolerances for the fabrication of the forks are less than a tenth of a mil. Figure 3 shows a typical wafer of forks. Statek formerly supplied these wafers of forks; now the wafers of forks are provided by Sandia National Laboratories' Prototype Processing Lab (PPL) in Bldg. 893.

Depending on the proposed application for the QDA, the required accuracy of measurement can range from $\pm 1 / 4 \mathrm{~g}$ for Environmental Sensing Devices (ESDs) to $\pm 10 \mu \mathrm{g}$ or less for navigational applications. The range of acceleration that this sensor is capable of measuring is 1 to $3 \mathrm{~g}$ up to $200 \mathrm{~g}$. Sensitivity to acceleration along axes other than the one being measured is desired to be $<1 \%$. The temperature range for some potential applications is $-55^{\circ} \mathrm{C}$ to $+85^{\circ} \mathrm{C}$.



Figure 3. Fork Wafer 


\section{The Problem}

The individual tuning forks in this assembly are very small (Figure 4) and very fragile; misalignments as small as 1 mil are large compared to the dimensions of the forks. Fork-to-fork or spacer-to-fork misalignments increase sensitivity to acceleration inputs in directions other than the one desired. Stress can also be induced in the assembly by poor control of the bond-joint dimensions. Such stresses may affect the temperature response and induce spurious oscillation modes.

\section{Early Assembly Techniques}

Early QDA cantilever assembly tooling made use of three locating masks: one for each tuning fork and one for the spacers. 'These masks were etched from 5-mil-thick beryllium copper by the Photofabricacion Shop, Div. 7413-1 (Figure 5). The dimensional tolerance that could be held on the parts-locating features and on alignment features of the tooling was typically 0.0005 in. (10\% of the material thickness).
The cantilevers were assembled individually by pairing two tuning forks from the saine wafer that were close to $1 \mathrm{kHz}$ apart in frequency, one at $\sim 70$ $\mathrm{kHz}$ and one at $\sim 71 \mathrm{kHz}$. Once the pair of forks was chosen, the fir't fork was placed in a fork-locating mask and polyimide adhesive was applied manually to the bonding areas of the fork. Then the spacerlocating mask was placed atop the first fork mask and the spacers emplaced. Polyimide was then applied to the bonding areas of the spacers. The last step was to position the second fork mask and emplace the second fork.

With this early fixturing it was difficult to produce QDA cantilevers with precise alignment. It was possible to accumulate a misalignment of as much as $0.0015 \mathrm{in}$. between the masks and the parts, and in the alignment of the masks to each other; this is large relative to the dimensions of the forks. Such a misalignment could severely degrade the sensur performance. Because polyimide was applied to the bonding areas manually with a pointed instrument, the results were very operator-dependent, making it difficult to achieve consistent results. The amount and placement of the polyimide are very important because uneven bond joints cause stress that can adversely affect QDA performance.

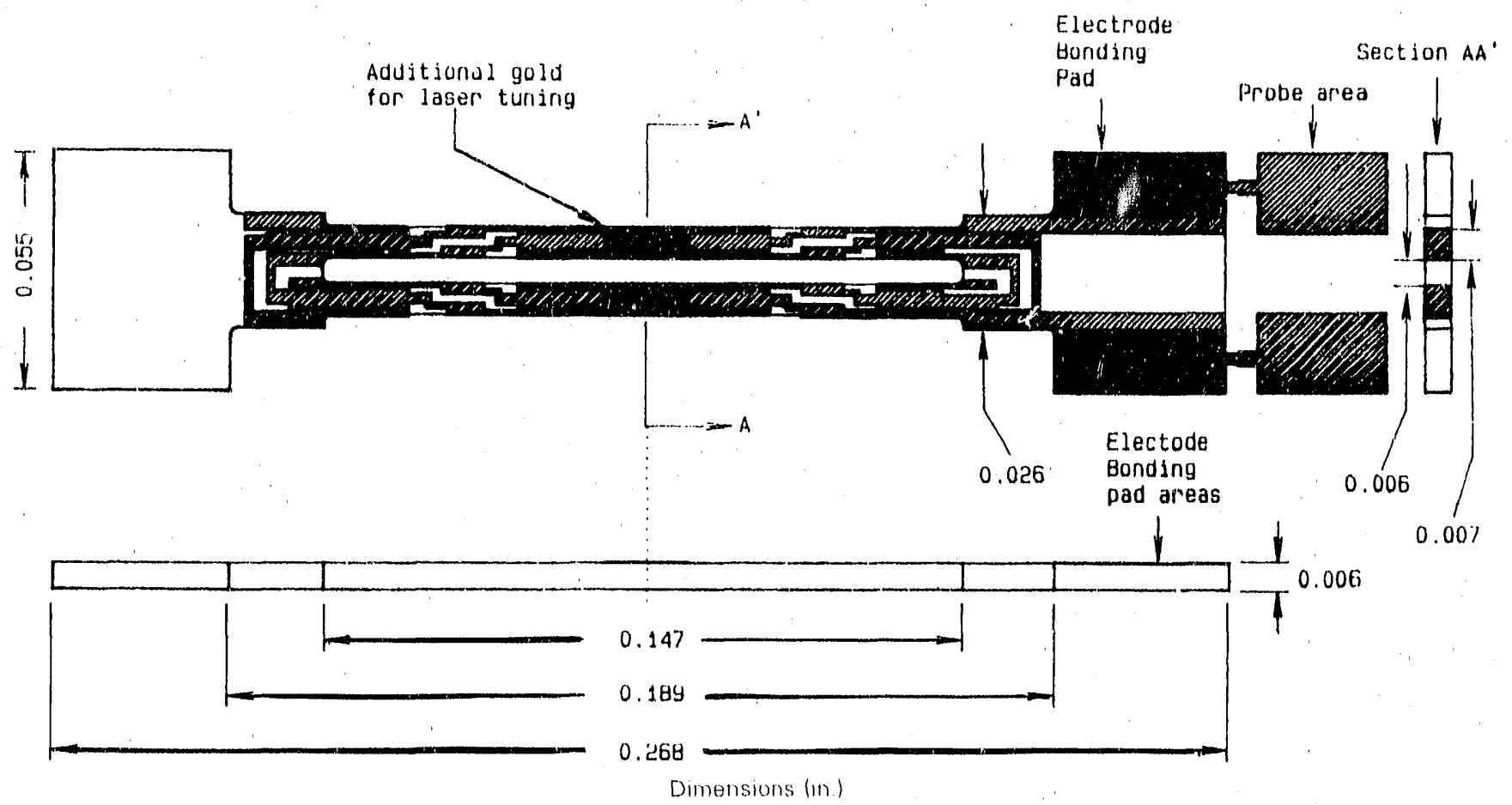

Figure 4. Double-Ended Tuning Fork 


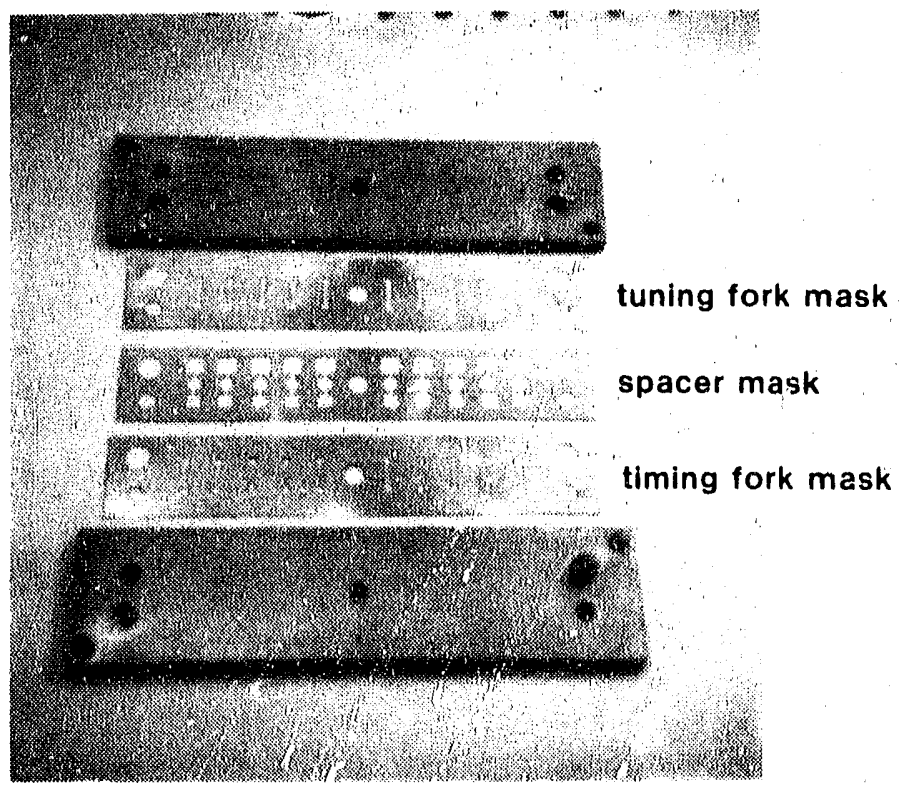

Figure 5. Early Cantilever Assembly Tooling

\section{Improved Single-Assembly Processes}

New fixturing was designed that addressed the tolerance buildun of the early tooling. Cushman Engineering, located in Albuquerque, fabricated the new fixture design for single QDDA rantilever assembly using a numerically controlled wire electron discharge machine (EDM). Cushrnan was chosen because of their ability to hold extremely tight tolerances $( \pm 0.0001 \mathrm{in}$.$) . The fixture consisted of three pieces: a$ base plate, a fork and spacer location nest, and a weight (Figure 6). The pieces of the fixture were assembled and aligned with dowel pins. The base plate had a 6 -mil recess the length of a tuning fork to ensure that the plane of the spacers remained parallel to the plane of the forks. The location nest aligned the tuning forks and spacers. Figure 7 shows a tuning fork in a location nest. The misalignment possible with this tooling has been reduced to $\sim 0.0006 \mathrm{in}$. using metal spacars and to $\sim 0.0003$ in. using quartz spacers. The weight places a slight force on the bonding areas to maintain an even pressure during polyimide curing. About 30 fixtures were made for various QDA cantilever designs.

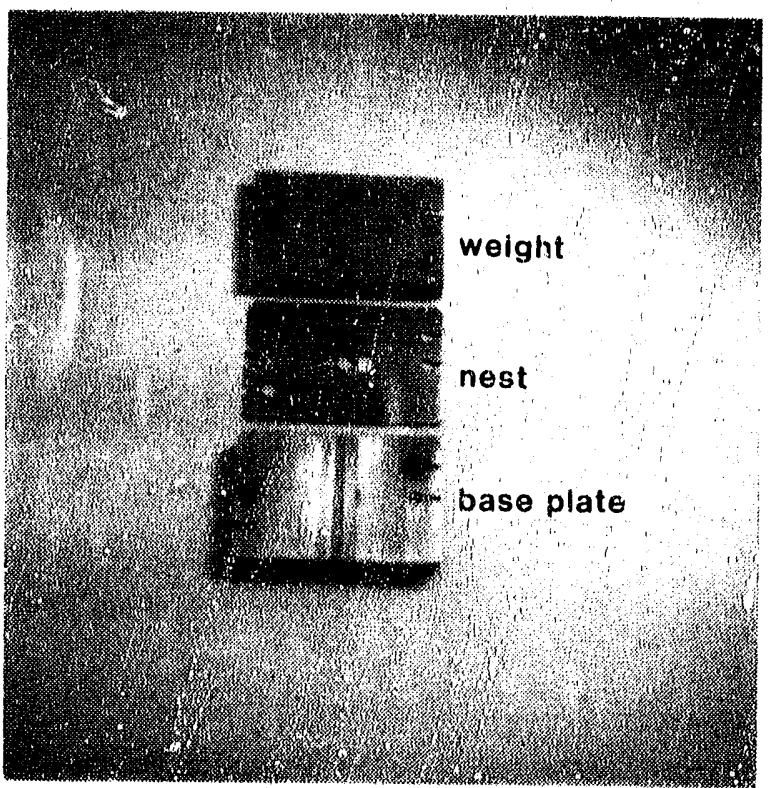

Figure 6. EDM Cantilever Assembly Tooling

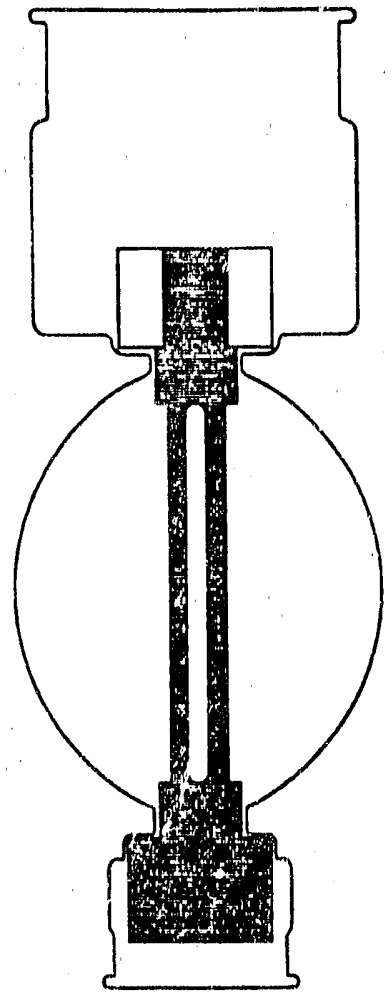

Figure 7. Fork Location Nest. 
A new polyimide adhesive application technique was also incorporated to improve the quality and repeatability of the bonds. The first approach used a metal applicator pin with a two-stage tip and a polyimide reservoir (Figure 8 ). The first stage regulated the size of the polyimide dot; the second stage regulated how far the first stage traveled into the reservoir. 'The applicator pin was lightly springloaded so as not to put excessive force on the quartz when polyimide was transferred to the tuning fork. The reservoir was a block of aluminum with a 5mil-deep groove. A doctor blade drawn across this groove precisely regulated the depth of the adhesive. The applicator pin was lowered into the reservoir and received a polyimicle dot of exact thickness and diameter. 'The pin was then applied to the bond area of the fork to transfer the polyimide. 'The design of the pin produced a dot of adhesive that, because it was higher in the center, did not trap a bubble of air when the parts were assembled (Figure 9). Figure 10 shows a comparison between a bond produced with the early technique and a bond produced with the applicator pin.

The resulting size and shape of the polyimide dot were very repeatable and were not operator-dependent (Figure 11.). One problem with applying polyimide in this manner was that it tended to dry out quickly in the reservoir because of evaporation of the polyimide vehicle atter being spread in a thin layer. This was exacerbated by the air flow of the clean bench where assembly took place. Often the polyimide formed "strings" when the applicator pins were withdrawn from the reservoir, a problem that was eliminated by redesigning the reservoir to allow a greater depth of polyimide and to include a cover to prevent the polyimide from drying. The tip of the applicator pin was also redesigned to accommodate the new reservoir (Figure 12).

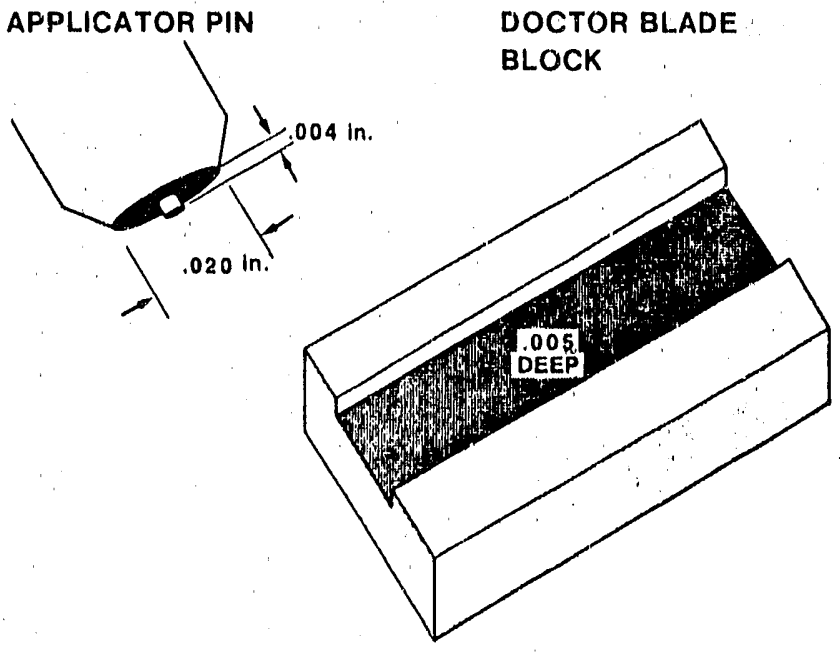

Figure 8. First Generation Applicator Pin
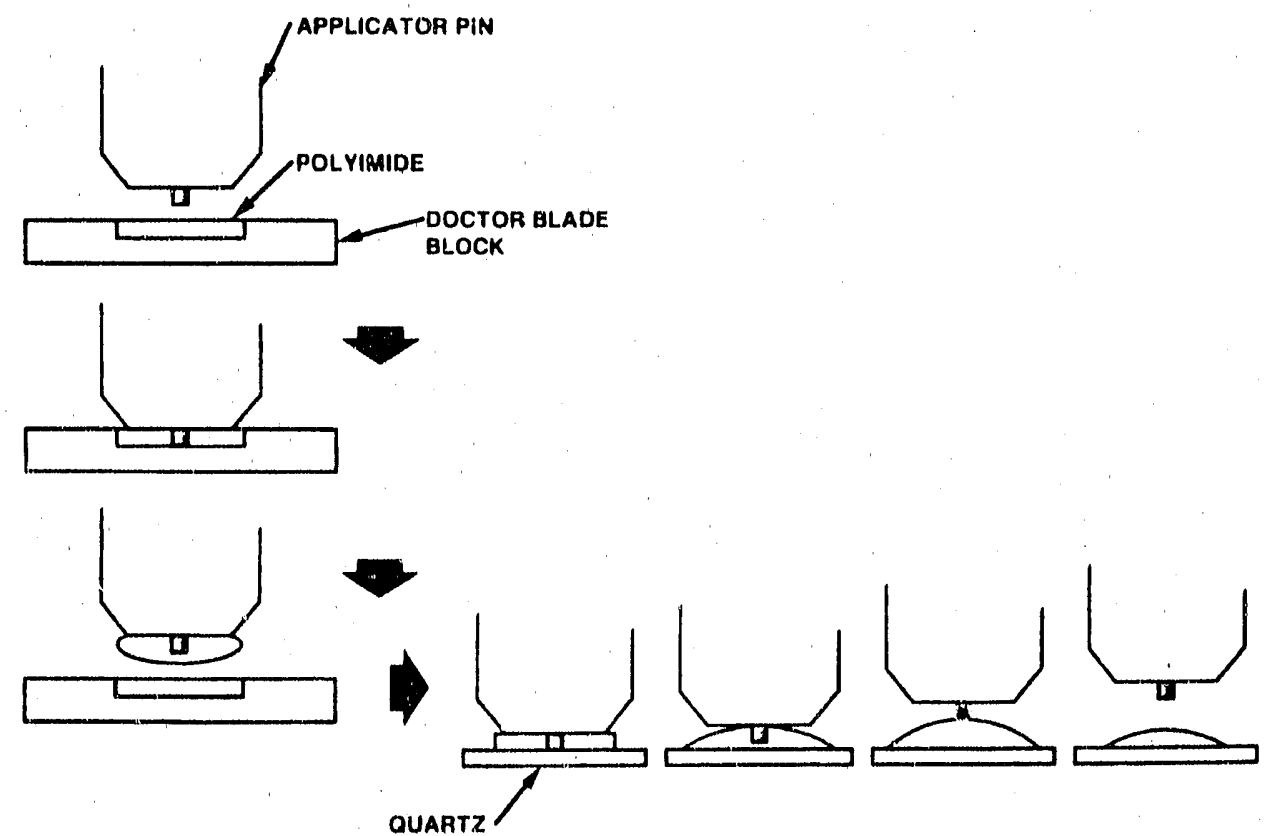

Figure 9. Polyimide Application Sequence 


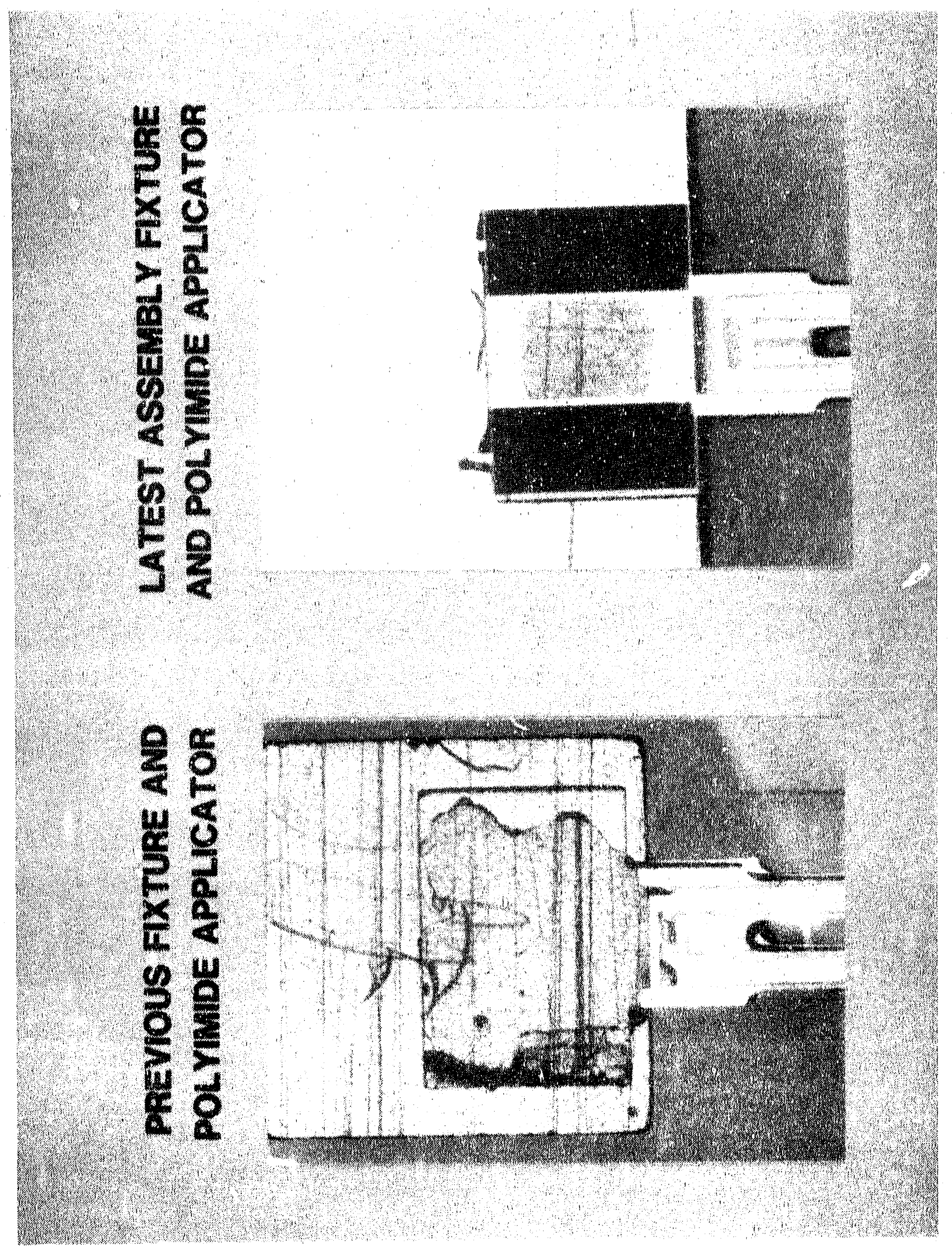

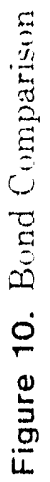




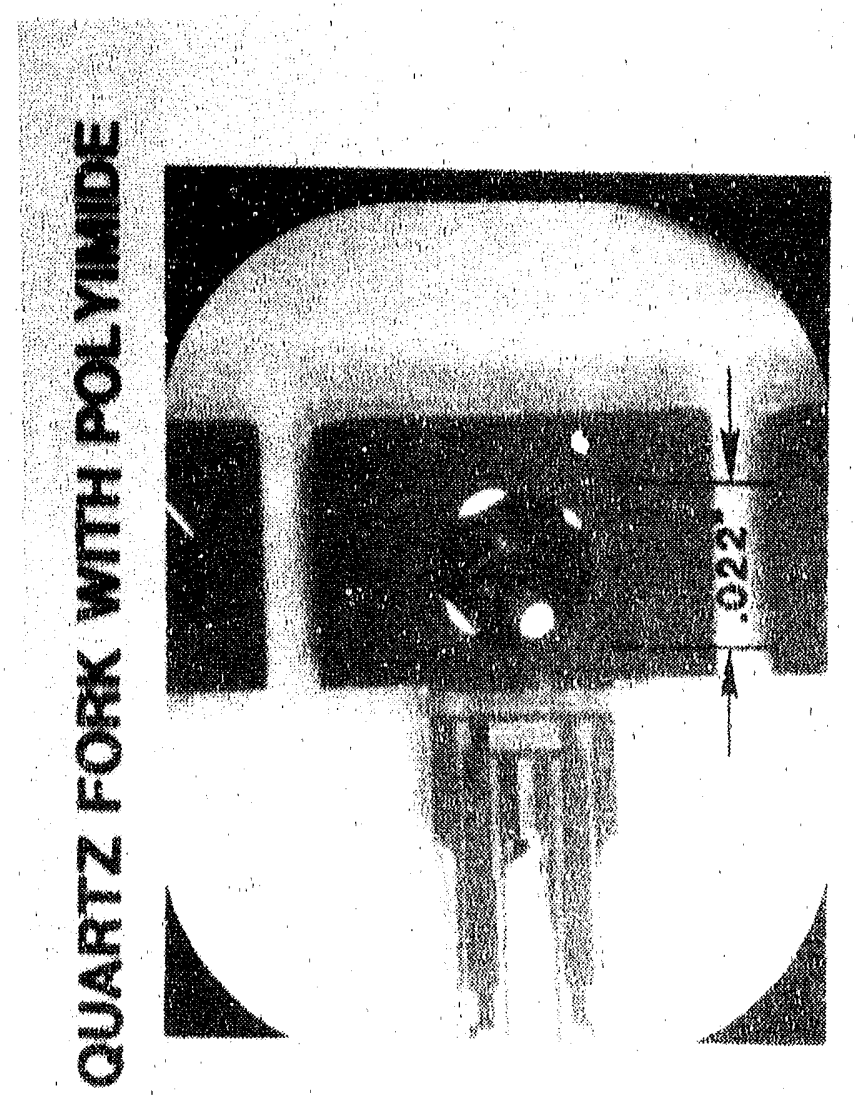

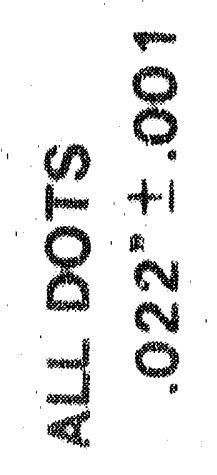
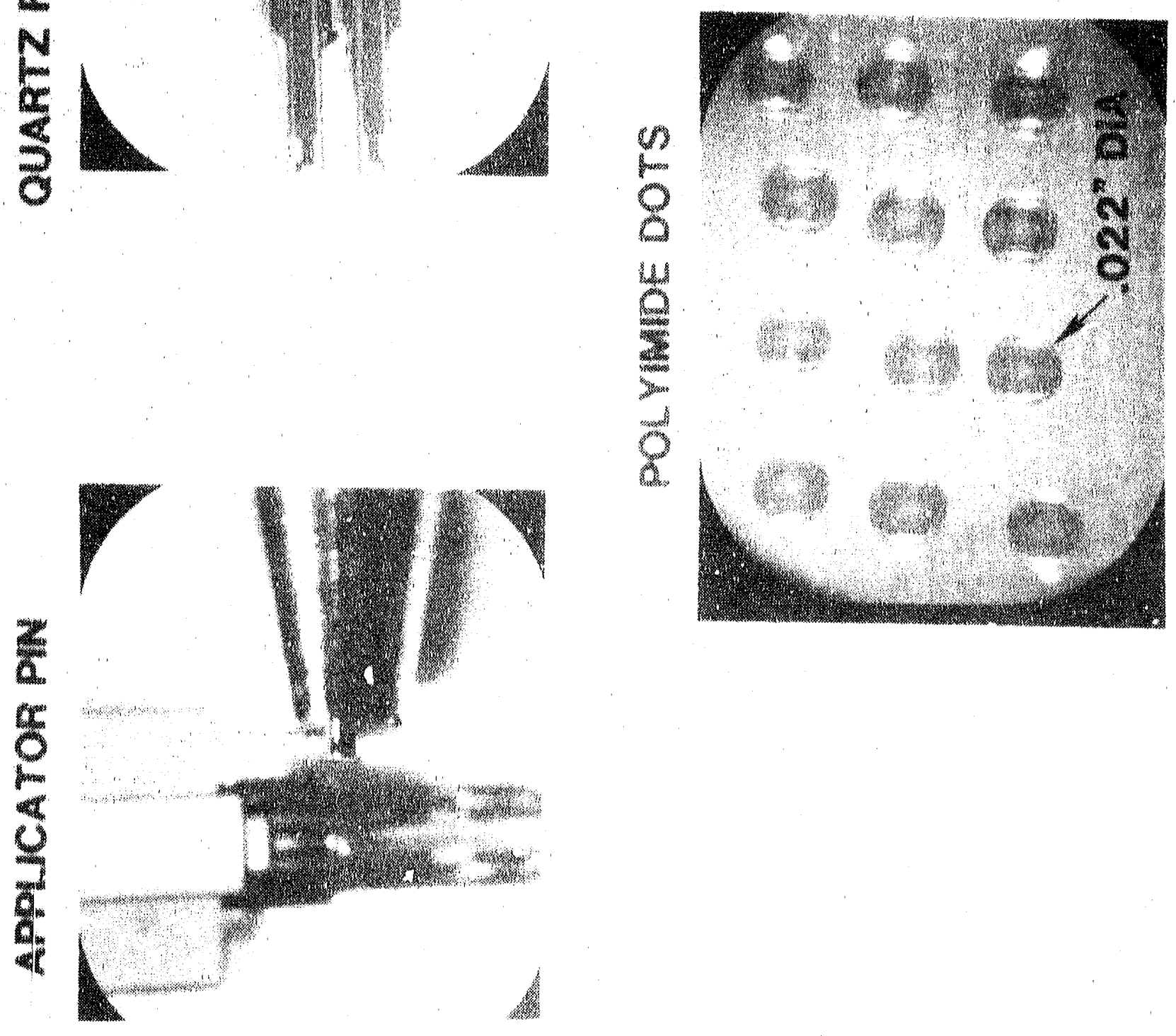

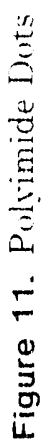



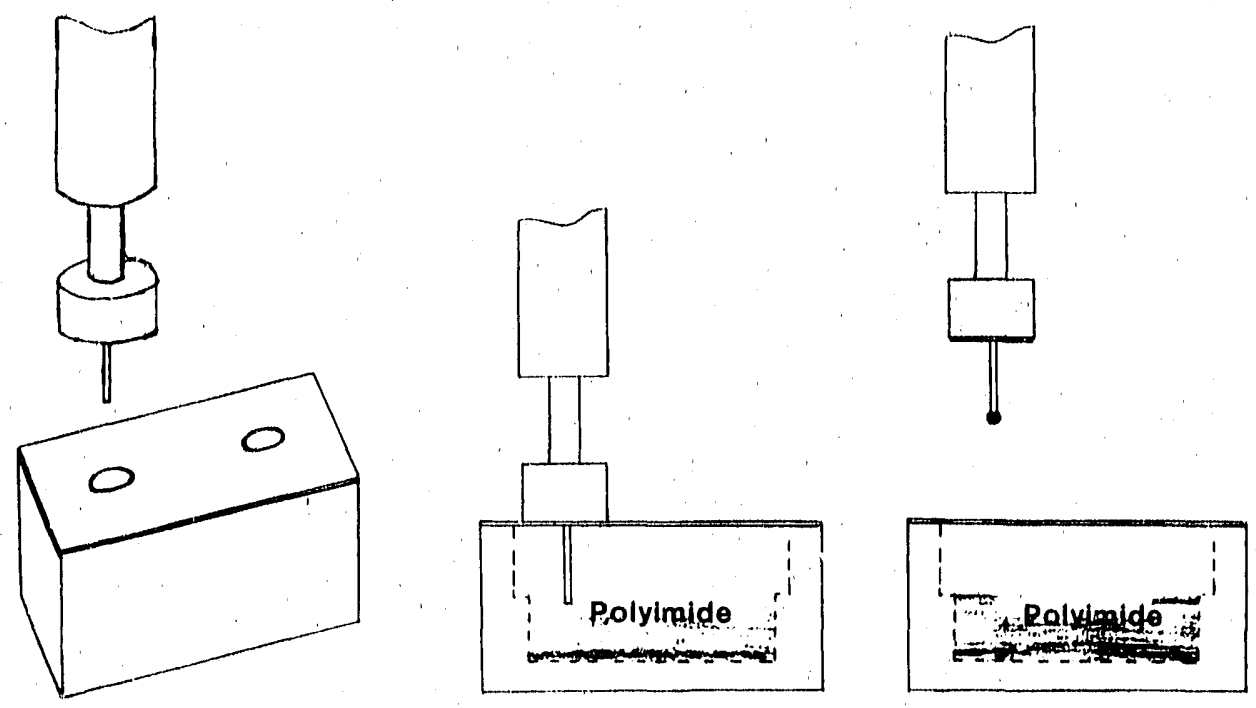

Figure 12. Second Generation Applicator Pin

Because asymmetric location of the adhesive results in uneven stress on the tuning fork, we turned our attention to the problem of precisely locating the polyimide dots on the part. A semiaut rmated polyimide applicator system was designed (Figure 13) consisting of two ball slide platforms actuated by low-pressure pneumatics. One platform slides horizontally, alternately positioning the parts and the polyimide reservoir under the applicator pins. The second slide moves the pins vertically to transfer the polyimide from the reservoir to the cantilever bond areas. Once the cantilever is assembled, the polyimide is oven-cured while still in the fixture.

A subtle problem was noted during development of the tooling. When the tuning forks are etched in the quartz wafers, the quartz etches anisotropically; thus the crystal facets on one side of the fork are larger than on the other. Waters were usually fabri- cated with forks of both frequencies on the same vafer and aligned in the same direction. Since the adhesive bond surfaces were on the same side of the wafer for both forks, after the forks were assembled into a cantilever, one fork had the bond side down and the other had the bond side up. This caused the large facet edge of the two forks to be on opposite sides of the cantilever assembly, which meant that the tooling offset one fork $\sim 0.0005$ in. relative to the other (Figure 14). To correct the problem we had two different wafers made, each containing forks of only one frequency, with the bond surface orientation of the $70-\mathrm{kHz}$ wafers the reverse of that of the $71-\mathrm{kHz}$ wafers.

The improved fixturing and the semiautomated polyimide applicator system resulted in an improved assembly alignment and bond uniformity. 







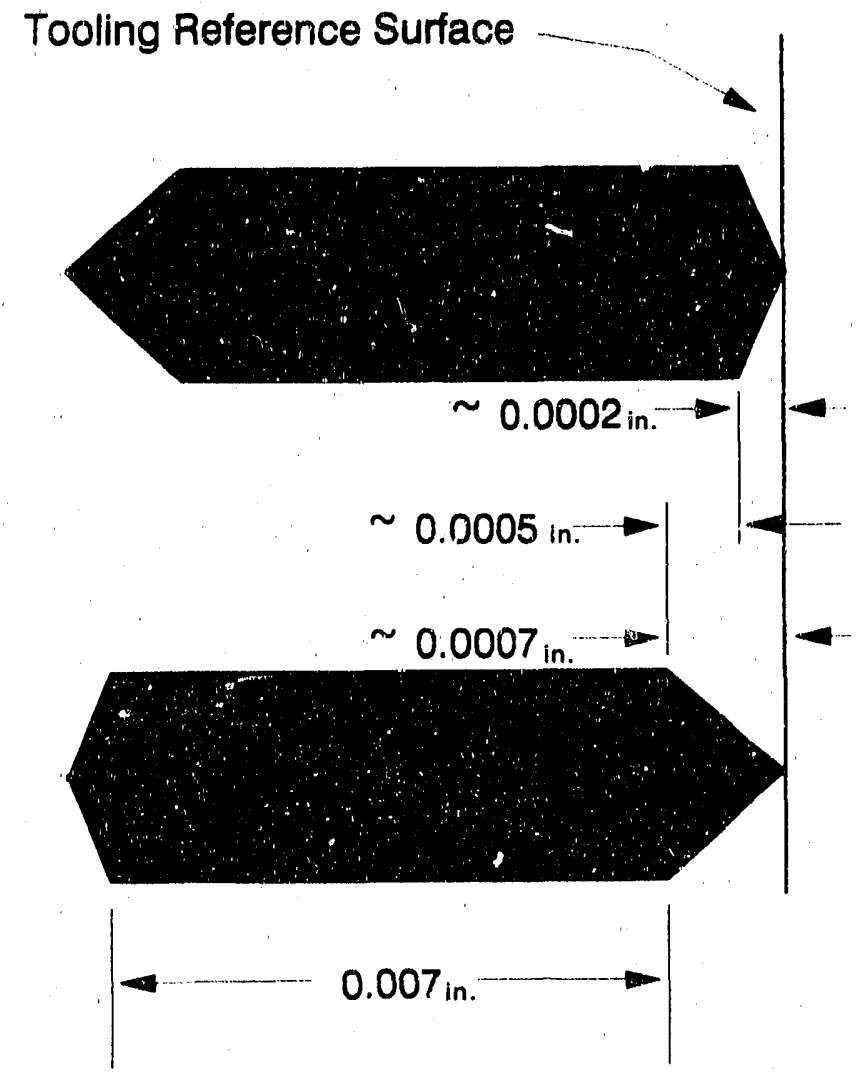

Figure 14. Fork Off'set (aused by Anisotropic Etching

\section{The Wafer Scale Cantilever Assembly}

We developed a second QDA cantilever assembly techniqu" called the "wafer scale assembly." In this process, 1 (1) wafers of forks instead of individual pairs of forks are assembled with a wafer of quartz spacers. In this way 20 cantilevers can be assembled simultaneomsly (Figure 15).* The advantages of this technique are excellent part alignment, simple tooling, multiple assembly of cantilevers, and exact thermal match between the quartz spacers and the forks. A water with forks having a nominal frequency of 70 $\mathrm{kHz}$, and a wafer with forks having a nominal frequency of $71 \mathrm{kHz}$ are assembled with the wafer of quartz spacers sandwiched between them. All three waters are positioned with the crystal facets aligned similarly.

\footnotetext{
*At present we are using wafers with 32 forks. Because of the interference between the center row of forks and the spacers for the outer rows, a 20-fork water was -'...signed and wit! soxn be placed in production?
}

This technique can hold very tight alignment tolerances because both the iork wafers and the quartz spacer wafer rely on the inherent ability of the photoetch process in quartz to maintain consistent part dimensions and location. Part and location tolerances on the wafers are typically less than a tenth of a mil. Alignment holes are included in the wafer design to permit the use of very simple tooling for assembly. The tooling consists of a metal block with two pins carefully machined to the dimensions of the alignment holes in the wafers.

There are two major disadvantages of wafer scale assembly. Each nonfunctional fork on a wafer causes a loss of the corresponding fork on the other wafer, and it is not possible to match all pairs of forks for a difference frequency of close to $1 \mathrm{kHz}$. To improve the practicality of wafer scile assembly, we must have wafers in which virtualiy all the forks are functional and most forks have frequencies within $150 \mathrm{~Hz}$ of the desired nominal frequency. (The practical tuning range of the cantilevers using a laser is $150 \mathrm{~Hz}$.) Experience to date with wafers from Statek has shown that fork-to-fork frequency can vary over a wafer by as much as $1 \mathrm{kHz}$; typically, it varies several hundred Hertz, Juergen Staurte, XECO, informed us that while he was at. Statek, he found that a frequency variability of $1{ }^{\prime \prime}$, or $700 \mathrm{~Hz}$, could be expected as the result of difficult.to-control variables in the normal commercial production process. ${ }^{1}$ Efforts are under way to reduce this variation by improved wafer processing at Sandia Laboratories and by adjusting the frequency of the forks while they are still on the wafer by means of adding gold to the tuning pad.

Wafer scale assembly has been prrformed using polyimide for bonding. A fixture was designed whereby a programmable $x-y$ table located the individual forks on the wafer under pairs of applicator pins that sequentially applied polyimide to the bonding areas of the forks from a reservoir (Figure 16).

Quartz spacers for cantilevers, because of their small size, do not have the mass required to give the cantilevers the desired sensitivity to acceleration. As a consequence, it was necessary to bond small weights to the free end of the assembly. (Figure 17). This was done in a location nest similar to that used for single-unit assembly. These weights are formed by using $\mathrm{EDM}$ machining to reduce the tolerance inherent with other forming methods. Since they are not adjacent to the active areas of the forks, the bonding procedure is not as critical. However, the weights must be carefully aligned with the axis of the cantilever and the mass of the weights is critical to the sensitivity of the assembly to acceleration. 

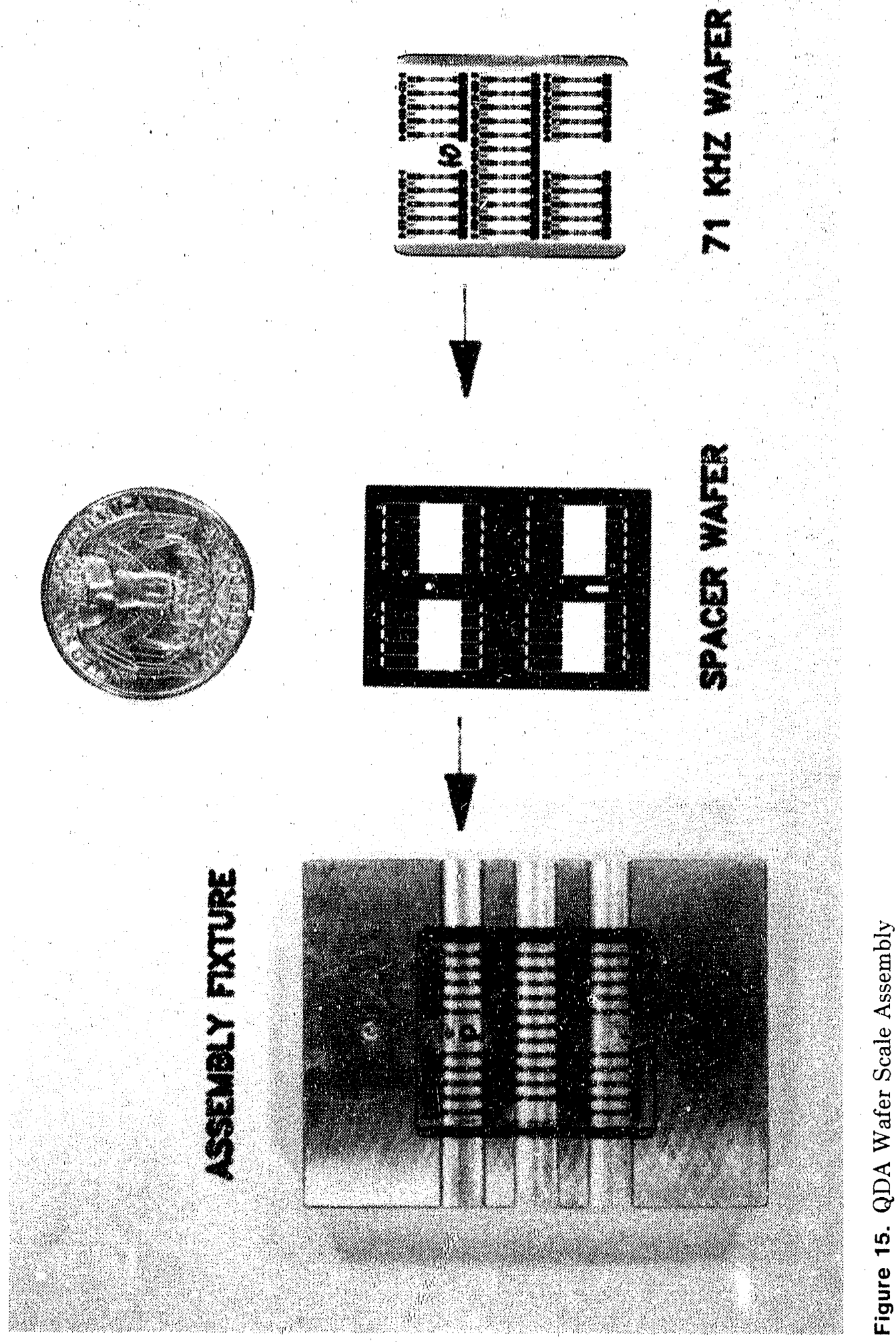


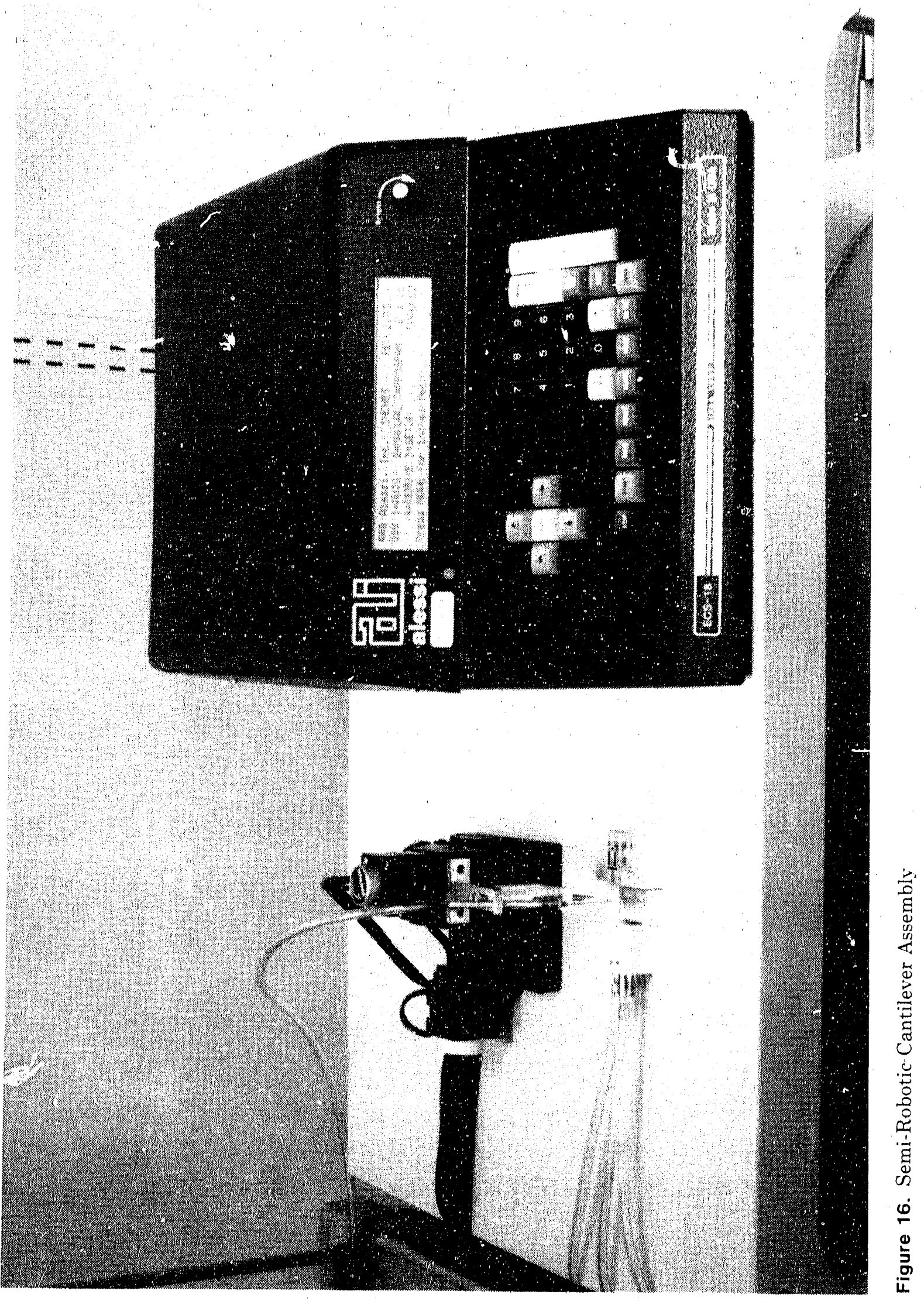




\section{Proof Mass \\ Weights}

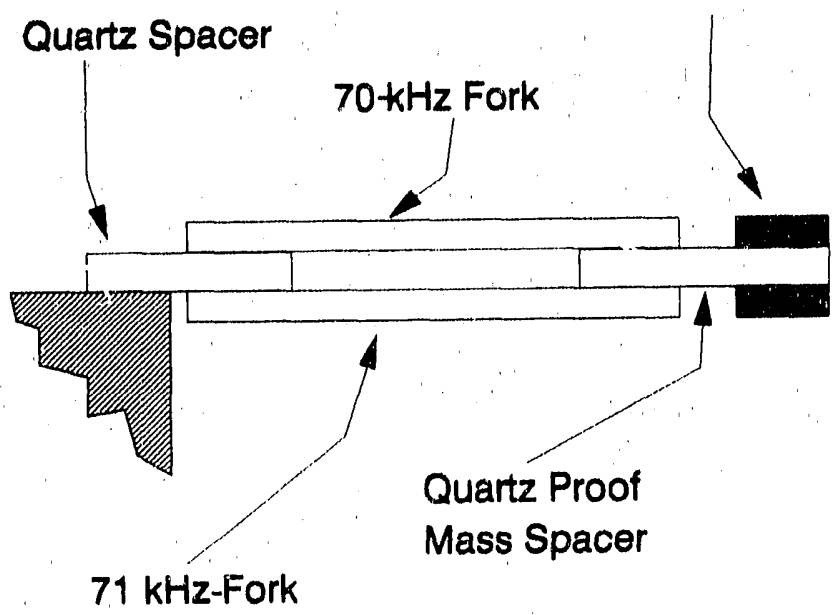

Figure 17. Quartz Spacer Assembly

\section{Eutectic Bunding}

Consideration of a eutectic to replace the polyimide adhesive for QDA cantilever bonding was promptea by several considerations. One was that, even with the improved application techniques, it was difficult to control precisely the thickness of polyimide bond joints. Finite-element studies, ${ }^{2}$ sponsored by Div. 2533 and performed by Dave Reedy of Div. 1.522, showed that uneven bond joints would adversely affect QDA performance. Another consideration was stress caused by the difference in the thermal coefficient of expansion between quartz and polyimide. The thermal coefficient of some eutectics more closely matches that of quartz. Less siress would be exerted on the quartz because of the thinner, more uniform joint thickness achieved by the use of eutectics. In addition, a solder bond could be annealed to further reduce stress.

The eutectic chosen was gold/germanium because it has a eutectic point of $356^{\circ} \mathrm{C}$, well above any subsequent processing temperature for the QDA package and also well below the transition point of quartz. (The ratio of gold to germanium at the eutectic point is $88 \%$ to $12 \%$ by weight.)

Initially we considered using preforms of the eutectic to perform the bonding. However, the preforms were very tiny and difficult to handle, the minimum thickness available was $0.001 \mathrm{in}$., and the dimensional tolerance was poor. Further, assuring that the preforms were precisely placed during cantilever assembly proved virtually impossible, which led to the consideration of sputtering the eutectic in place. The eutectic bond studies required for this approach were performed by Paul Vianco, Div. 1831.

Sputtering of the eutectic on wafers of goldplated spacers was attractive since the location and thickness could be controlled very precisely. Aperture masks controlled the location and dimensions of the applied eutectic, and the time and rate of the sputtering controlled the thickness. However, Paul Viacico found that oxidation of the germanium at the surface of the eutectic inhibited bonding. Bond joints could be obtained, but not reliably or repeai bly. This experience led to development of the present technique.

The present technique consists of evaporating or sputtering alternate layers of gold and germanium onto the spacers, using aperture masks as before to control location and dimensions. The layer thicknesses used reflect the proportions of gold and germanium at che eutectic point. The germanium layer is sandwiched between layers of gold, thus preventing oxidation during heatup. At a few degrees below the eutectic temperature, the germanium starts to diffuse into the gold to form the eutectic solder, which then flows when the melting point is reached. Bonds produced by this technique were very uniform and repeatable. Bond thicknesses as low as $0.5 \mu$ m have been achieved. Presently, we are using $2-\mu \mathrm{m}$-thick bonds although, at this thickness, the assembly and bonding must be done under clean-room conditions. This is because ordinary air-borne particles are of the same size as the thickness of the bond joint. Particles on the surface can keep the substrates apart anc prevent bonding.

Development continues on the eutectic bonding process. Cantilevers have been made with both single cantilever tooling and wafer scale tooling. In both cases it was necessary to apply a small pressure to the bond joints to assure intimaie contact during bonding. The assembly procedure was greatly simplified by elimination of the polyimide appl.cation process. With either single assembly or wafer scale assembly, we need only stack the parts in a fixture, apply a small force on the bond joint, and then heat the parts slightly above the eutectic reflow temperature.

\section{Transfer of Technology to GEND}

We intend to transfer a defined set of processes and tooling to GEND that will permit production assemblies to have the same performance as exhibited by Sandia-fabricated prototypes. With this intention, we have periodically reported our progress to GEND 
and have sent them tooling for single and wafer scale cantilever assembly and for semiatutomated polyimide application. Demonstrations of assembly techniques and training were also provided, 'The transfer will continue as we develop improved tooling and procedures.

\section{Conclusions}

The QDA cantilevers assembled using processes discussed in this report show a marked reduction in part misalignment and bond joint variations. Further, assembly has been greatly simplified, particularly through the use of wafer scale assembly and eutectic bonding. Limited test results indicate that the temperature response has also improved. Testing on a large number of samples will be required to establish and confirm the actual degree of QDA improvement. A further benefit of this effort is that the processes developed can be adapted to the assembly of other miniature compcnents.

\section{Further Work}

Our development effort to irruprove the cantilever assembly highlighted several other areas also requir- ing further work. One is to improve the fabrication of the wafers of forks. Our goal is to produce wafers with all forks having acceptable electrical characteristics and a much-reduced frequency variability-a goal we are pursuing in the PPL. To back up these efforts, a wafer slew tester was designed and is being built to fully characterize each fork while it is still on the wafer. Also in the design stage is equipment to individually tune each fork, while it is still on the wafer, by using an intense gold beam source to deposit gold on the tuning pad area of the fork tines. These two instruments will complement the wafer improvement work and should help provide better wafers for the assembly process.

\section{References}

${ }^{1}$ J. Staudte, XECO, to C. T. Cebert, Sandia National Laboratories, Albuquerque, NM, June 1989, private coin. munication.

"E. D. Reedy to W. J. Kass, "Finite-Element Analysis of a Quartz Digital Accelerometer," Sandia National Laboratories, Albuquerque, NM, unpublished report. 

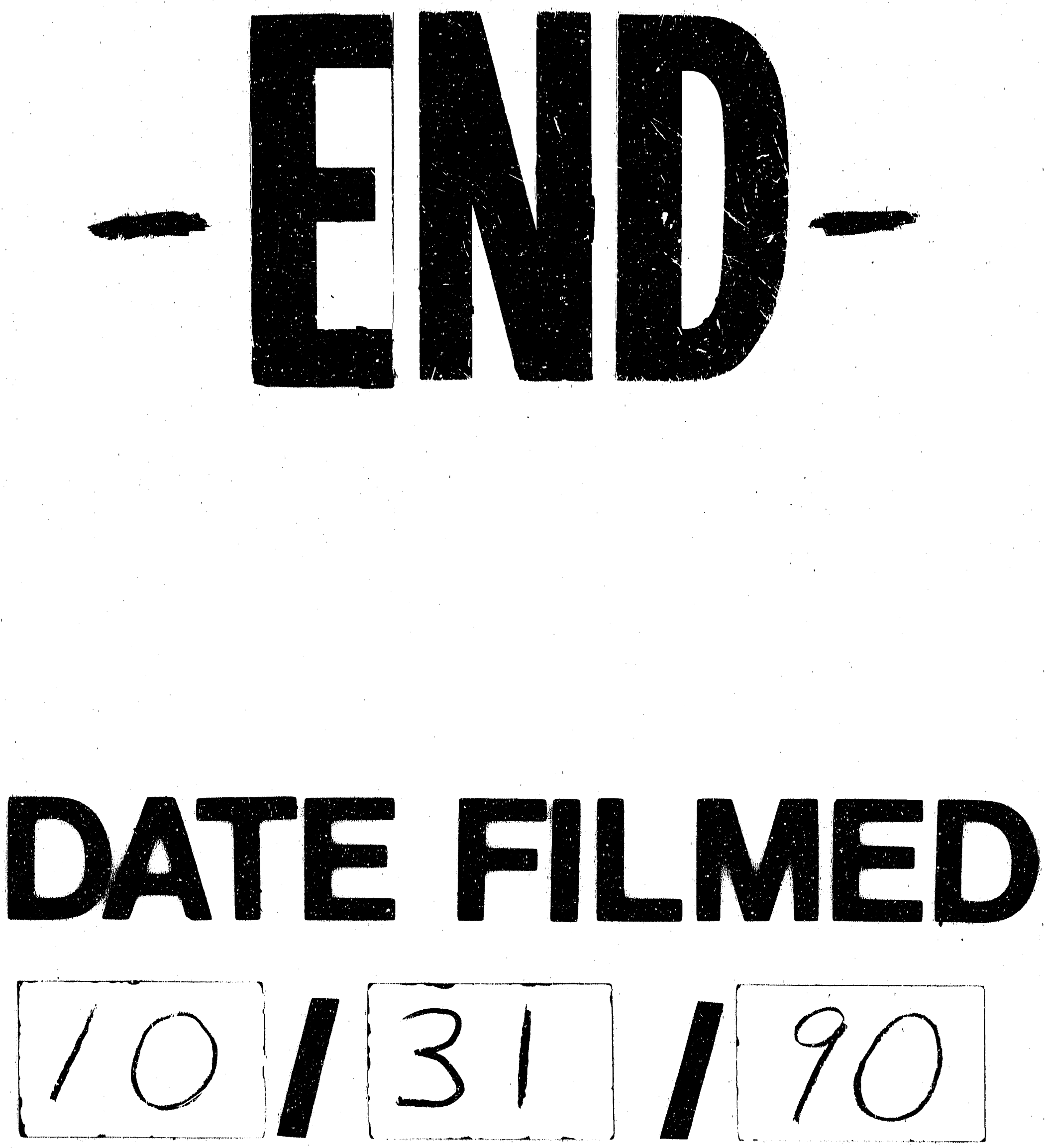
